\title{
Sprinting, Change of Direction Ability and Horizontal Jump Performance in Youth Runners According to Gender
}

\author{
by \\ Javier Yanci', Asier Los Arcos ${ }^{1}$ Daniel Castillo², Jesús Cámara ${ }^{1}$
}

The purpose of the study was to assess straight sprinting, change of direction ability and horizontal jump performance in youth runners according to age and gender. Two hundred and fifty-five youth runners (116 boys and 139 girls) participated in this study. The athletes were divided according to their age into five groups: under 8 yr (U8), under $10 \mathrm{yr}$ (U10), under $12 \mathrm{yr}$ (U12), under $14 \mathrm{yr}$ (U14) and under $16 \mathrm{yr}$ (U16). Significant differences $(p<0.01)$ were found between U8 and U10 in the $5 \mathrm{~m}$ sprint $(d=1.22), 505$ agility test $(505, d=0.96)$, modified agility test (MAT, $d=1.43)$, horizontal countermovement jump (HCMJ, $d=1.06)$ and arm swing HCMJ (HCMJAS, $d=1.44)$; between U10 and U12 in the $505(d=0.39), M A T(d=0.74), H C M J(d=0.96)$ and HCMJAS $(d=0.75)$; and between U12 and U14 in $5 \mathrm{~m}(d=0.84), \mathrm{HCMJ}(d=0.88)$ and HCMJAS $(d=0.79)$. However, no significant differences ( $p>$ $0.05, d=0.29-1.17)$ between U14 and U16 were observed in any of the tests. With regard to age and gender, in U8 and U10 groups there were no significant differences ( $p>0.05, d=0.02-0.76)$ between boys and girls in any test. However, in U12 and U14 groups, significant gender differences $(p<0.05, d=0.85-1.24)$ were found in the MAT. Likewise, the boys obtained better results than girls in the horizontal jump tests $(p<0.05, d=1.01-1.26)$. After the classification by age, some differences were observed between both genders, depending on the fitness variable evaluated.

Key words: field test, maturity, agility, strength, acceleration, athletes.

\section{Introduction}

Physical activity and fitness play a significant role in prevention of overweight and obesity in children and adolescents (Ortega et al., 2013, 2015). Some recommendations indicate that children should get involved in $60 \mathrm{~min}$ of moderate to vigorous physical activity at least three days a week, in order to prevent overweight and obesity during puberty (Lätt et al., 2015). It has been also reported that the fitness level of children and teenagers has declined over the last three decades (Catley and Tomkinson, 2013). To solve this issue, some researchers have studied physical capacities in children and adolescents using field condition tests (Lopes et al., 2011; Malina et al., 2004; Vescovi et al., Yanci et al., 2013). Yet, these tests seem to be more age-related and moreover, young athletes are familiar with them. It seems that a high level of fitness during childhood and adolescence may be associated with better health in adulthood and indeed with lower total and central adiposity at this age and later in life (Ortega et al., 2015).

Several distances of straight line sprinting, change of direction ability (CODA) and horizontal jump tests have been carried out in children and adolescents engaged in team sports (Meylan et al., 2014; Spencer et al., 2011) and individual sports (Eisenmann and Malina, 2003). Furthermore, physical fitness performance has been examined over a 10-12 year period that included puberty and adolescence in various sports (Castro-Pinero et al., 2010; Vescovi et al., 2011). Physical fitness performance is considered a relevant key to assess the level of physical conditioning in different sports in youth athletes and is a powerful health marker in childhood and

1 - Faculty of Education and Sport, University of the Basque Country, UPV/EHU, Vitoria-Gasteiz, Spain.

${ }^{2}$ Faculty of Health Sciences, University Isabel I, Burgos, Spain 
adolescence (Ortega et al., 2015). There is, as yet, no consensus about the number and the kind of field tests that young athletes should perform.

Some studies have examined physical characteristics in youth runners (Eisenmann and Malina, 2003; Jaric et al., 2002). The differences in agility and explosive-power between genders were attenuated before 13 years of age in youth runners. However, during the adolescent growth spurt, differences between the gender emerged as a result of a continued increase in males and a plateau in females (Eisenmann and Malina, 2003). Since we did not find studies analyzing acceleration capacity, CODA and horizontal jump ability in young runners, it seemed that it would be interesting to assess fitness performance at a younger age in this population. Likewise, in spite of the fact that age and gender seem to be highly related to physical fitness performance in childhood and adolescence in team sports (Mendez-Villanueva et al., 2011; Sekulic et al., 2013; Spencer et al., 2011), more scientific studies are necessary regarding these physical capacities in individual sports, particularly in youth runners.

Therefore, the purposes of the study were first, to assess sprinting, change of direction ability and horizontal jump performance in youth runners, secondly, to determine the differences according to age and gender, and thirdly, to analyze the relationships among these abilities.

\section{Methods}

\section{Participants}

Two hundred and fifty-five youth runners (116 boys and 139 girls) from an athletic training school participated in this study. Athletes were divided according to their age into five groups: under 8 yr $(\mathrm{U} 8, \mathrm{n}=75)$, under $10 \mathrm{yr}(\mathrm{U} 10, \mathrm{n}=68)$, under $12 \mathrm{yr}(\mathrm{U} 12, \mathrm{n}=48)$, under $14 \mathrm{yr}(\mathrm{U} 14, \mathrm{n}=41)$ and under $16 \mathrm{yr}(\mathrm{U} 16, \mathrm{n}=23)$ (Table 1). They all had training experience of at least one year (range $=1-7$ years). All athletes performed regular endurance, sprint and specific running training ranging from 2 to 4 days per week. All parents or tutors of participants gave their written informed consent before their inclusion in the study in accordance with the Declaration of Helsinki (2013). They were also informed of their right to voluntarily withdraw from the study at any given time. The study was approved by both the Basque
Country ethics committee and the Hiru Herri club to which the athletes belonged.

\section{Procedures}

In this study, we examined sprinting, CODA, and horizontal jumping abilities in youth athletes on two consecutive days. Tests were carried out during the competitive season and all athletes were required to attend the exercisetesting session on two separate occasions. Physical performance was determined by testing performance qualities that are usually evaluated in athletes (Hoare and Warr, 2000; Maulder and Cronin, 2005; Yanci et al., 2013). Prior to the testing session, the researchers provided the participants with graphic and direct instructions about how to successfully perform the test. Two test sessions were performed to practice the tests and to ensure that the participants performed both tests correctly.

During the first test session, the runners were informed about the testing protocol and anthropometric measurements were taken followed by a $15 \mathrm{~m}$ straight sprint test. The modified agility test (MAT) and the 505 agility test (505) were also performed during the first session to determine CODA. The second testing session took place $48 \mathrm{~h}$ after the first session. During the second session runners performed the horizontal jump (HJ). Always before testing, a warm-up was performed and it consisted of $5 \mathrm{~min}$ self-paced low-intensity running, skipping exercises, strides, two $15 \mathrm{~m}$ sprints with and without changes of direction and two vertical and horizontal jumps.

\section{Measures}

Straight Sprint test

Each athlete performed an acceleration test consisting of three maximal sprints of $15 \mathrm{~m}$, with a 2 min rest period between each sprint, giving enough time to walk back to the start and wait for another turn as previously described by Yanci et al. (2013). The participants were placed at $0.5 \mathrm{~m}$ behind the starting point, and began when they felt ready. Split time at $5 \mathrm{~m}$ and the time to cover the $15 \mathrm{~m}$ were measured. Time was recorded using photocell gates (Microgate $^{\mathrm{TM}}$ Polifemo, Bolzano, Italy). The timer was automatically activated as the participants passed the first gate at the $0 \mathrm{~m}$ mark.

Change of direction ability (CODA)

Modified agility test (MAT): Athletes 
completed the protocol previously described by Yanci et al. (2013). All participants performed the test three times with at least 3 min rest between each performance. The total distance covered was $20 \mathrm{~m}$. A photocell (Microgate $^{\mathrm{TM}}$ Polifemo, Bolzano, Italy) was used to record the time.

505 agility test (505): Athletes sprinted forward to a line $5 \mathrm{~m}$ ahead and pivoted $180^{\circ}$ before returning to the start position (Hoare and Warr, 2000; Sheppard and Young, 2006). A photocell (Microgate ${ }^{\mathrm{TM}}$ Polifemo, Bolzano, Italy) located over the start/finish line was used to record the time. Time measurement started and finished when the subject crossed the line between the tripods.

Horizontal jump (HJ) tests

Following the procedures previously proposed by Maulder and Cronin (2005) and Yanci et al. (2014), runners performed 3 horizontal countermovement jumps (HCMJ), and 3 arm swing countermovement jumps (HCMJAS). Recovery time between jumps was $20 \mathrm{~s}$ and recovery time between each type of the jump was 4 min.

\section{Statistical Analysis}

The results are presented as mean \pm standard deviation (SD). All the variables were normally distributed and satisfied the equality of variance according to the Shapiro-Wilk and Levene's tests, respectively. Only the maximum score for each test was included in the data analysis. Independent paired t-tests were used to determine if any significant differences existed between the boys' and girls' groups. One-way ANOVA was used to determine if any significant differences existed between the five age groups (U8-U16) and a two (male and female) $x$ five (different age groups) ANOVA to explore sex differences. Bonferroni post-hoc analysis was applied to any set of hypothesis tests. Practical significance was assessed by calculating Cohen's d effect size (Cohen, 1998). Effect sizes (d) of above 0.8 , between 0.8 and 0.5 , between 0.5 and 0.2 and lower than 0.2 were considered as large, moderate, small, and trivial, respectively. Pearson product-moment correlation coefficients $(\mathrm{r})$ and simple regression models were calculated to determine the relationships among the variables obtained from the sprint, CODA and horizontal jump testing. The magnitude of correlation between test measures was assessed with the following thresholds: $<0.1$, trivial; $=0.1-0.3$, small; $<0.3-0.5$, moderate; $<0.5-0.7$, large; $<0.7-0.9$, very large; and $<0.9-1.0$, almost perfect (Hopkins et al., 2009). Data analysis was performed using the Statistical Package for Social Sciences (version 20.0 for Windows, SPSSTM Inc, Chicago, IL, USA) for Windows. Statistical significance was set at $p<$ 0.05 .

\section{Results}

The results of the physical performance in sprint, CODA and $\mathrm{HJ}$ tests of the total sample, boys and girls are described in Table 2. The CV of all the physical tests were between 1.9 and $4.9 \%$. No significant differences $(p>0.05, \mathrm{~d}<0.10$, trivial) were observed between boys and girls in any of the variables.

The results of the physical tests according to the age groups between U8 and U16 are presented in Table 3. Significant differences were found between U8 and U10 considering the $5 \mathrm{~m}$ sprint, 505, MAT, HCMJ and HCMJAS; between $\mathrm{U} 10$ and $\mathrm{U} 12$ in the 505, MAT, HCMJ and HCMJAS; and between U12 and U14 in the $5 \mathrm{~m}$ sprint, HCMJ and HCMJAS. However, no significant differences between U14 and U16 were observed in any of the physical tests. Only a large $(\mathrm{d}=1.17)$ and moderate $(\mathrm{d}=0.78)$ effect size in the $15 \mathrm{~m}$ sprint and MAT, respectively, were found between U14 and U16.

With regard to age and gender (Table 4 ), no significant differences $(p>0.05, d=0.02-0.76)$ were observed between boys and girls in the U8 and U10 in any test. However, in U12 and U14 groups, significant gender differences $(p<0.05, \mathrm{~d}$ $=0.85-1.24)$ were observed in the MAT. Likewise, U16 boys obtained better results than U16 girls in the horizontal jump tests ( $p<0.05, \mathrm{~d}=1.01-1.26)$.

The relationship between the straight sprint, CODA and $\mathrm{HJ}$ test of the total sample is described in Table 2. Significant correlations were found among all of the tests in all athletes. A negative correlation $\left(r=-0.711, R^{2}=0.529, p<0.01\right.$, very large, $\mathrm{y}=1.978-0.345 \mathrm{x}+0.071)$ was found between the $5 \mathrm{~m}$ sprint test and HCMJAS. Furthermore, the MAT values correlated negatively with the HCMJ $\left(\mathrm{r}=-0.782, \mathrm{R}^{2}=0.529, p\right.$ $<0.01$, very large, $y=1.978-0.345 x+0.071)$. 


\begin{tabular}{|c|c|c|c|c|c|}
\hline \multicolumn{6}{|c|}{ Table 1} \\
\hline & $\begin{array}{l}\text { Age } \\
(\mathrm{yr})\end{array}$ & $\begin{array}{c}\text { Body mass } \\
(\mathrm{kg})\end{array}$ & $\begin{array}{l}\text { Body height } \\
(\mathrm{m})\end{array}$ & $\begin{array}{c}\text { BMI } \\
\left(\mathrm{kg} / \mathrm{m}^{2}\right)\end{array}$ & $\begin{array}{c}\text { Training experience } \\
(\mathrm{yr})\end{array}$ \\
\hline Boys $(n=116)$ & $10.22(2.73)$ & 37.54 (12.14) & $1.37(0.28)$ & $17.76(2.06)$ & $2.25(1.81)$ \\
\hline Girls (n=139) & $10.43(2.62)$ & 37.94 (11.38) & $1.40(0.30)$ & $17.86(2.64)$ & $2.47(2.03)$ \\
\hline U8 $(n=75)$ & $7.33(0.55)$ & $26.38(4.48)$ & $1.09(0.15)$ & 16.34 (1.63) & $1.25(0.44)$ \\
\hline $\mathrm{U} 10(\mathrm{n}=68)$ & $9.47(0.68)$ & 34.38 (6.85) & $1.39(0.08)$ & $17.41(2.23)$ & $2.13(1.12)$ \\
\hline $\mathrm{U} 12(\mathrm{n}=48)$ & $11.27(0.71)$ & $41.81(8.15)$ & $1.52(0.42)$ & $18.36(2.46)$ & $2.65(1.80)$ \\
\hline $\mathrm{U} 14(\mathrm{n}=41)$ & $13.44(0.59)$ & $49.78(7.78)$ & $1.60(0.07)$ & $19.40(2.12)$ & $3.15(2.30)$ \\
\hline $\mathrm{U} 16(\mathrm{n}=23)$ & $15.22(0.60)$ & $54.70(5.96)$ & $1.66(0.08)$ & $19.90(1.71)$ & $4.74(3.26)$ \\
\hline Sample $(n=255)$ & $10.34(2.67)$ & $37.76(11.71)$ & $1.39(0.29)$ & $17.81(2.39)$ & $2.37(1.94)$ \\
\hline$U 8=$ & $\begin{array}{l}8 \text { years g } \\
\quad U 14=\imath\end{array}$ & $\begin{array}{l}\text { Uean }(S D, \text { sto } \\
\text { U10 = under } \\
14 \text { years gro }\end{array}$ & $\begin{array}{l}\text { deviation), } \\
\text { ears group, } \\
16=\text { under }\end{array}$ & $\begin{array}{l}=\text { body mo } \\
=\text { under } 12 \\
\text { ars group }\end{array}$ & $\begin{array}{l}\text { dex, } \\
\text { s group, }\end{array}$ \\
\hline
\end{tabular}

\begin{tabular}{|lcccc|}
\hline \multicolumn{5}{c}{ Table 2} \\
Straight sprint, change of direction ability and horizontal jump test performance \\
for total sample $(n=255)$, boys $(n=116)$ and girls $(n=139)$.
\end{tabular}

\begin{tabular}{|c|c|c|c|c|c|c|c|c|c|}
\hline \multicolumn{10}{|c|}{$\begin{array}{r}\text { Table } 3 \\
\text { Physical test results according to age groups between U8 and U16 }\end{array}$} \\
\hline & U8 & U10 & U12 & U14 & U16 & U8-U10 d & $\begin{array}{c}\text { U10-U12 } \\
\mathrm{d}\end{array}$ & U12-U14 d & U14-U16 d \\
\hline \multicolumn{10}{|l|}{ Sprint (s) } \\
\hline $\begin{array}{r}15 \mathrm{~m} \\
\mathrm{CODA}(\mathrm{s})\end{array}$ & $\begin{array}{l}1.35 \\
(0.07) \\
2.82 \\
(0.52)\end{array}$ & $\begin{array}{l}1.26 \\
(0.10) \\
2.85 \\
(0.39)\end{array}$ & $\begin{array}{l}1.24 \\
(0.09) \\
2.67 \\
(0.40)\end{array}$ & $\begin{array}{l}1.16 \\
(0.08) \\
2.76 \\
(0.25)\end{array}$ & $\begin{array}{l}1.13 \\
(0.08) \\
2.47 \\
(0.31)\end{array}$ & $\begin{array}{l}1.22 \\
(* *) \\
0.05 \\
\text { (NS) }\end{array}$ & $\begin{array}{l}0.18 \\
\text { (NS) } \\
0.44 \\
\text { (NS) }\end{array}$ & $\begin{array}{l}0.84 \\
(* *) \\
0.21 \\
(\mathrm{NS})\end{array}$ & $\begin{array}{l}0.43 \\
\text { (NS) } \\
1.17 \\
\text { (NS) }\end{array}$ \\
\hline 505 & $\begin{array}{l}3.13 \\
(0.26) \\
8.96 \\
(0.69)\end{array}$ & $\begin{array}{l}2.87 \\
(0.27) \\
7.99 \\
(1.15)\end{array}$ & $\begin{array}{l}2.77 \\
(0.16) \\
7.13 \\
(0.49)\end{array}$ & $\begin{array}{l}2.75 \\
(0.24) \\
6.79 \\
(0.59)\end{array}$ & $\begin{array}{c}2.66 \\
(0.15) \\
6.32 \\
(0.47)\end{array}$ & $\begin{array}{l}0.96 \\
(* *) \\
1.43 \\
(* *)\end{array}$ & $\begin{array}{l}0.39 \\
(* *) \\
0.74 \\
(* *) \\
(* *)\end{array}$ & $\begin{array}{l}0.07 \\
\text { (NS) } \\
0.70 \\
\text { (NS) }\end{array}$ & $\begin{array}{l}0.39 \\
\text { (NS) } \\
0.78 \\
\text { (NS) }\end{array}$ \\
\hline \multicolumn{10}{|c|}{ Horizontal jump (m) } \\
\hline HCMJ & $\begin{array}{c}1.04 \\
(0.11) \\
1.21 \\
(0.15) \\
\end{array}$ & $\begin{array}{r}1.15 \\
(0.16) \\
1.43 \\
(0.19) \\
\end{array}$ & $\begin{array}{c}1.31 \\
(0.16) \\
1.56 \\
(0.21) \\
\end{array}$ & $\begin{array}{c}1.45 \\
(0.20) \\
1.73 \\
(0.26) \\
\end{array}$ & $\begin{array}{c}1.51 \\
(0.13) \\
1.86 \\
(0.24) \\
\end{array}$ & $\begin{array}{l}1.06 \\
(* *) \\
1.44 \\
(* *) \\
(* *)\end{array}$ & $\begin{array}{l}0.96 \\
\left(^{* * *}\right) \\
0.75 \\
\left(^{*}\right) \\
\end{array}$ & $\begin{array}{l}0.88 \\
(* *) \\
0.79 \\
(* *) \\
\end{array}$ & $\begin{array}{r}0.29 \\
\text { (NS) } \\
0.52 \\
\text { (NS) } \\
\end{array}$ \\
\hline & $\begin{array}{l}\text { ta are } \\
\text { under } \\
\text { under } \\
\text { MAT } \\
\text { Sign }\end{array}$ & $\begin{array}{l}\text { sente } \\
\text { years } \\
\text { years } \\
\text { nodifi } \\
\text { ICM] } \\
\text { int di } \\
\text { IS }=1\end{array}$ & $\begin{array}{l}\text { mear } \\
\text { up, } \\
\text { up, } \\
\text { gility } \\
=\text { hor } \\
\text { nce ( } \\
\text { gnific }\end{array}$ & $\begin{array}{l}D, \text { st } \\
=u n \\
A= \\
t ; H C \\
\text { ital a } \\
<0.0 \\
\text { diffe }\end{array}$ & 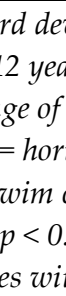 & $\begin{array}{l}\text { ion), U } \\
\text { roup, } \\
\text { ection al } \\
\text { atal cout } \\
\text { ntermov } \\
\text { with th } \\
\text { he previ }\end{array}$ & 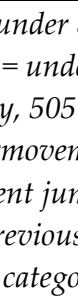 & $\begin{array}{l}\text { rs grou } \\
\text { years } g \\
5 \text { agility } \\
\text { jump, } \\
\text { gory. }\end{array}$ & \\
\hline
\end{tabular}


Table 4

Physical test results according age groups (U8-U16) and gender (boys and girls)

\begin{tabular}{|c|c|c|c|c|c|c|c|c|c|}
\hline & U8 & U10 & U12 & U14 & U16 & $\begin{array}{c}\text { U8-U10 } \\
\text { d }\end{array}$ & $\begin{array}{c}\text { U10-U12 } \\
\text { d }\end{array}$ & U12-U14 d & U14-U16 d \\
\hline \multicolumn{10}{|c|}{ Sprint $5 \mathrm{~m} \mathrm{(s)}$} \\
\hline Boys & $\begin{array}{c}1.35 \\
(0.08)\end{array}$ & $\begin{array}{c}1.25 \\
(0.09)\end{array}$ & $\begin{array}{c}1.23 \\
(0.07)\end{array}$ & $\begin{array}{c}1.18 \\
(0.10)\end{array}$ & $\begin{array}{c}1.11 \\
(0.06)\end{array}$ & $\begin{array}{l}1.27 \\
(* *)\end{array}$ & $\begin{array}{l}0.23 \\
(\mathrm{NS})\end{array}$ & $\begin{array}{l}0.77 \\
(\mathrm{NS})\end{array}$ & $\begin{array}{l}0.70 \\
\text { (NS) }\end{array}$ \\
\hline Girls & $\begin{array}{c}1.34 \\
(0.07)\end{array}$ & $\begin{array}{c}1.26 \\
(0.10)\end{array}$ & $\begin{array}{c}1.24 \\
(0.10)\end{array}$ & $\begin{array}{c}1.15 \\
(0.06)\end{array}$ & $\begin{array}{c}1.15 \\
(0.09)\end{array}$ & $\begin{array}{l}1.16 \\
(* *)\end{array}$ & $\begin{array}{l}0.15 \\
\text { (NS) }\end{array}$ & $\begin{array}{l}0.88 \\
\text { (NS) }\end{array}$ & $\begin{array}{l}0.03 \\
\text { (NS) }\end{array}$ \\
\hline $\mathrm{d}$ & 0.23 & 0.02 & 0.10 & 0.25 & 0.71 & - & - & - & - \\
\hline \multicolumn{10}{|c|}{ Sprint 15 m (s) } \\
\hline Boys & $\begin{array}{c}2.85 \\
(0.55)\end{array}$ & $\begin{array}{c}2.86 \\
(0.41)\end{array}$ & $\begin{array}{c}2.71 \\
(0.40)\end{array}$ & $\begin{array}{c}2.81 \\
(0.29)\end{array}$ & $\begin{array}{c}2.49 \\
(0.28)\end{array}$ & $\begin{array}{l}0.02 \\
\text { (NS) }\end{array}$ & $\begin{array}{l}0.36 \\
\text { (NS) }\end{array}$ & $\begin{array}{l}0.26 \\
\text { (NS) }\end{array}$ & $\begin{array}{l}1.11 \\
\text { (NS) }\end{array}$ \\
\hline Girls & $\begin{array}{c}2.80 \\
(0.49)\end{array}$ & $\begin{array}{c}2.84 \\
(0.39)\end{array}$ & $\begin{array}{c}2.65 \\
(0.41)\end{array}$ & $\begin{array}{c}2.72 \\
(0.21)\end{array}$ & $\begin{array}{c}2.45 \\
(0.35)\end{array}$ & $\begin{array}{l}0.08 \\
\text { (NS) }\end{array}$ & $\begin{array}{l}0.48 \\
\text { (NS) }\end{array}$ & $\begin{array}{l}0.18 \\
\text { (NS) }\end{array}$ & $\begin{array}{l}1.31 \\
\text { (NS) }\end{array}$ \\
\hline $\mathrm{d}$ & 0.09 & 0.06 & 0.16 & 0.32 & 0.14 & - & - & - & - \\
\hline \multicolumn{10}{|c|}{505 agility test (s) } \\
\hline Boys & $\begin{array}{c}3.14 \\
(0.31)\end{array}$ & $\begin{array}{c}2.92 \\
(0.37)\end{array}$ & $\begin{array}{c}2.76 \\
(0.17)\end{array}$ & $\begin{array}{c}2.68 \\
(0.17)\end{array}$ & $\begin{array}{c}2.62 \\
(0.16)\end{array}$ & $\begin{array}{c}0.70 \\
\left({ }^{*}\right)\end{array}$ & $\begin{array}{l}0.42 \\
(\mathrm{NS})\end{array}$ & $\begin{array}{l}0.46 \\
\text { (NS) }\end{array}$ & $\begin{array}{l}0.37 \\
\text { (NS) }\end{array}$ \\
\hline Girls & $\begin{array}{c}3.12 \\
(0.21)\end{array}$ & $\begin{array}{c}2.84 \\
(0.16)\end{array}$ & $\begin{array}{c}2.77 \\
(0.16)\end{array}$ & $\begin{array}{c}2.80 \\
(0.27)\end{array}$ & $\begin{array}{c}2.71 \\
(0.14)\end{array}$ & $\begin{array}{l}1.33 \\
(* *)\end{array}$ & $\begin{array}{l}0.43 \\
\text { (NS) }\end{array}$ & $\begin{array}{l}0.22 \\
\text { (NS) }\end{array}$ & $\begin{array}{l}0.34 \\
\text { (NS) }\end{array}$ \\
\hline d & 0.06 & 0.21 & 0.03 & 0.72 & 0.56 & - & - & - & - \\
\hline \multicolumn{10}{|l|}{ MAT (s) } \\
\hline Boys & $\begin{array}{c}8.96 \\
(0.70)\end{array}$ & $\begin{array}{c}8.02 \\
(1.61)\end{array}$ & $\begin{array}{c}6.96 \\
(0.35)\end{array}$ & $\begin{array}{c}6.40 \\
(0.47)\end{array}$ & $\begin{array}{c}6.20 \\
(0.54)\end{array}$ & $\begin{array}{l}1.33 \\
(* *)\end{array}$ & $\begin{array}{l}0.66 \\
(* *)\end{array}$ & $\begin{array}{l}1.61 \\
\text { (NS) }\end{array}$ & $\begin{array}{l}0.43 \\
\text { (NS) }\end{array}$ \\
\hline Girls & $\begin{array}{c}8.97 \\
(0.68)\end{array}$ & $\begin{array}{c}7.96 \\
(0.70)\end{array}$ & $\begin{array}{c}7.25 \\
(0.55) \#\end{array}$ & $\begin{array}{c}6.98 \\
(0.56) \# \#\end{array}$ & $\begin{array}{c}6.47 \\
(0.35)\end{array}$ & $\begin{array}{l}1.49 \\
(* *)\end{array}$ & $\begin{array}{l}1.01 \\
(* *)\end{array}$ & $\begin{array}{l}0.49 \\
\text { (NS) }\end{array}$ & $\begin{array}{l}0.91 \\
\text { (NS) }\end{array}$ \\
\hline $\mathrm{d}$ & 0.02 & 0.04 & 0.85 & 1.24 & 0.50 & - & - & - & - \\
\hline \multicolumn{10}{|c|}{$\mathrm{HCMJ}(\mathrm{m})$} \\
\hline Boys & $\begin{array}{c}1.00 \\
(0.11)\end{array}$ & $\begin{array}{c}1.13 \\
(0.19)\end{array}$ & $\begin{array}{c}1.33 \\
(0.17)\end{array}$ & $\begin{array}{c}1.51 \\
(0.25)\end{array}$ & $\begin{array}{c}1.58 \\
(0.13)\end{array}$ & $\begin{array}{c}1.32 \\
\left({ }^{*}\right)\end{array}$ & $\begin{array}{l}1.03 \\
\left({ }^{* *}\right)\end{array}$ & $\begin{array}{c}1.05 \\
\left({ }^{*}\right)\end{array}$ & $\begin{array}{l}0.29 \\
\text { (NS) }\end{array}$ \\
\hline Girls & $\begin{array}{c}1.08 \\
(0.10)\end{array}$ & $\begin{array}{c}1.17 \\
(0.13)\end{array}$ & $\begin{array}{c}1.29 \\
(0.16)\end{array}$ & $\begin{array}{c}1.42 \\
(0.17)\end{array}$ & $\begin{array}{c}1.42 \\
(0.08) \# \#\end{array}$ & $\begin{array}{c}0.93 \\
(* *)\end{array}$ & $\begin{array}{l}0.92 \\
\left({ }^{* *}\right)\end{array}$ & $\begin{array}{l}0.81 \\
(* *)\end{array}$ & $\begin{array}{l}0.02 \\
\text { (NS) }\end{array}$ \\
\hline $\mathrm{d}$ & 0.76 & 0.18 & 0.23 & 0.37 & 1.26 & - & - & - & - \\
\hline \multicolumn{10}{|c|}{ HCMJAS (m) } \\
\hline Boys & $\begin{array}{c}1.18 \\
(0.14)\end{array}$ & $\begin{array}{c}1.48 \\
(0.21)\end{array}$ & $\begin{array}{c}1.61 \\
(0.21)\end{array}$ & $\begin{array}{c}1.81 \\
(0.35)\end{array}$ & $\begin{array}{c}1.98 \\
(0.25)\end{array}$ & $\begin{array}{l}2.17 \\
(* *)\end{array}$ & $\begin{array}{l}0.62 \\
\text { (NS) }\end{array}$ & $\begin{array}{l}1.00 \\
(\mathrm{NS})\end{array}$ & $\begin{array}{l}0.48 \\
\text { (NS) }\end{array}$ \\
\hline Girls & $\begin{array}{c}1.24 \\
(0.16)\end{array}$ & $\begin{array}{c}1.37 \\
(0.14)\end{array}$ & $\begin{array}{c}1.53 \\
(0.21)\end{array}$ & $\begin{array}{c}1.69 \\
(0.20)\end{array}$ & $\begin{array}{c}1.73 \\
(0.15) \#\end{array}$ & $\begin{array}{l}0.83 \\
\text { (NS) }\end{array}$ & $\begin{array}{l}1.21 \\
\text { (NS) }\end{array}$ & $\begin{array}{l}0.73 \\
(* *)\end{array}$ & $\begin{array}{l}0.21 \\
\text { (NS) }\end{array}$ \\
\hline $\mathrm{d}$ & 0.45 & 0.51 & 0.37 & 0.37 & 1.01 & - & - & - & - \\
\hline
\end{tabular}

Data are presented as mean (SD, standard deviation), U8 = under 8 years group, $U 10=$ under 10 years group, $U 12=$ under 12 years group, U14 = under 14 years group, U16 $=$ under 16 years group, $C O D A=$ change of direction ability, $505=505$ agility test,

$M A T=$ modified agility test; HCMJ = horizontal countermovement jump,

HCMJAS = horizontal arm swim countermovement jump.

Significant difference $\left({ }^{* *} p<0.01,{ }^{*} p<0.05\right)$ with the previous category.

Significant difference (\# $p<0.01, \# p<0.05$ ) between boys and girls.

$N S=$ no significant differences with the previous category 


\begin{tabular}{|c|c|c|c|c|}
\hline \multicolumn{5}{|c|}{$\begin{array}{l}\text { Table } 5 \\
\text { Relationship between straight sprint ( } 5 \text { and } 15 \mathrm{~m} \text { ), change } \\
\text { of direction ability (505 and MAT) and horizontal jump (HCMJ } \\
\text { and HCMJAS) tests in all youth athletes }\end{array}$} \\
\hline & $505(\mathrm{~s})$ & MAT (s) & $\operatorname{HCMJ}(\mathrm{m})$ & $\operatorname{HCMJAS}(\mathrm{m})$ \\
\hline Sprint $5 \mathrm{~m}(\mathrm{~s})$ & $0.617^{* *}$ & $0.709^{* *}$ & $-0.727^{* *}$ & $-0.711^{* *}$ \\
\hline Sprint $15 \mathrm{~m}$ (s) & $0.312^{* *}$ & $0.289^{* *}$ & $-0.229^{* *}$ & $-0.171^{*}$ \\
\hline $505(\mathrm{~s})$ & - & $0.764^{* *}$ & $-0.600^{* * *}$ & $-0.617^{\text {*** }}$ \\
\hline MAT (s) & - & - & $-0.762^{* *}$ & $-0.782^{* *}$ \\
\hline \multicolumn{5}{|c|}{$\begin{array}{c}505=505 \text { agility test, } M A T=\text { modified agility test; } \\
\text { HCMJ = horizontal countermovement jump, } \\
\text { HCMJAS = horizontal arm swim countermovement jump. } \\
\text { Significant correlation }\left({ }^{* *} p<0.01\right) \text {. }\end{array}$} \\
\hline
\end{tabular}

\section{Discussion}

The objectives of this study were threefold, to assess sprinting, change of direction ability and horizontal jump performance in youth runners, to determine the differences according to age and gender and to analyze the relationship among these abilities. Even though the influence of age and gender on physical performance has been widely analyzed in many sports (Doré et al., 2008; Jaric et al., 2002; Kugler and Janshen, 2010; Malina et al., 2004), to our knowledge no study has determined sprinting capacity, CODA and horizontal jump capacity in an athletic training school in youth athletes. This information could help coaches develop specific training programs adapted to the physical characteristics of the athletes.

Our results show significant differences between U8-U10 and U12-U14 in the $5 \mathrm{~m}$ sprint and between U8-U10 and U10-U12 in CODA (505 and MAT). Moreover, the HCMJ and HCMJAS also differed between U8-U10, U10-U12 and between U12-U14. These results coincide with a previous study where differences in strength between particular ages were shown to be maintained until the age of $18 \mathrm{yr}$ (Taeymans et al., 2009). In spite of the observed differences across ages in participants under 14 , no significant differences were found between U14-U16 in any of the physical tests. Only a large $(\mathrm{d}=1.17)$ and moderate $(\mathrm{d}=0.78)$ effect size was found between U14 and U16 in youth runners in the $15 \mathrm{~m}$ and MAT. Considering, on the one hand, that peak height velocity occurs at the age of $13 \mathrm{yr}$ and that from the age of 15 to 16 an increase of $20 \%$ has been observed in maximal power output in a group of boys (Kugler and Janshen, 2010), and on the other hand, that peak fiber size does not plateau until the age of 16 years (Eisenmann and Malina, 2003), we expected significant differences in jumping, sprinting and CODA in the participants between U14 and U16. Furthermore, taking into account that chronological age itself exerts a positive influence on short term power output (Armstrong et al., 2001) and that the tests performed in this study are positively related to this characteristic, we cannot explain why the observed differences in younger ages were not 
maintained above 14 yr. Further studies are required in order to gain a deeper insight into the study of the evolution of short term power performance across ages above $14 \mathrm{yr}$. The lack of significant differences observed in the present study in sprinting, jumping capacity and CODA in athletes over 14 suggests the need to train short term power output specifically above this age.

We did not find statistical $(p=0.05)$ nor practical differences $(\mathrm{d}=$ trivial $)$ in the physical performance tests between boys and girls (Table 2). However, after the classification by age, some differences were observed between both genders. Therefore, as previous studies have reported (Eisenmann and Malina, 2003; Yanci et al., 2014a), the maturation process may affect fitness variables differently and, in consequence, differences between both genders should be expected. Specifically, while the practical differences in acceleration capacity (i.e. $5 \mathrm{~m}$ and $15 \mathrm{~m})$ were trivial or small in all age groups except for U16 group in the $5 \mathrm{~m}$ test $(\mathrm{d}=0.71)$ and CODA (i.e. 505 and MAT), moderate practical differences were found between genders in both tests for the older two groups (i. e., U14 and U16) and in the MAT for the U12 age group (Table 4). In general, the differences in acceleration and sprinting capacities between boys and girls seem more relevant in older than in younger athletes (Amusa et al., 2010; Eisenmann and Malina, 2003; Lam and Schiller, 2001; Yanci et al., 2014b) also in CODA (Eisenmann and Malina, 2003; McKenzie et al., 2002; Yanci et al., 2014a), independently of the impact of the test characteristics (Brughelli et al., 2008; Chaouachi et al., 2012; Yanci et al., 2014a) and these differences between the genders are emphasized during the adolescent growth spurt (Eisenmann and Malina, 2003). In contrast to these two abilities, moderate practical differences were found between genders in horizontal jump performance (i. e., HCMJ and HCMJAS) independently of age. This result again implies that the differences between boys and girls at different ages may also vary depending on the fitness variable tested. Only few studies have analyzed horizontal force (Eisenmann and Malina, 2003) in athletes, and compared results according to gender, even though it is an important variable during the accelerative and later stages of sprinting (Kugler and Janshen, 2010; Mero, 1998). In youth long distance runners,
Eisenmann and Malina (2003) found differences between genders in standing long jump performance in athletes over 13 years of age. Yet, these differences were not observed in younger athletes. Even though their results differ with ours, although not dramatically, we suggest that the differences between boys and girls in acceleration and horizontal jump abilities were not equal for all of the age groups.

The association values among the performance variables varied depending on the tests (Table 5). The correlation between CODA and $\mathrm{HJ}$ performance was positive and large-very large for all tests $(r=0.60-0.78)$. Conversely, the relationships between the performance in the acceleration and in the rest of the capacities, positive in all cases, were considerably lower with respect to the $15 \mathrm{~m}$ test $(\mathrm{r}=0.17-0.31$, small $)$ in comparison with the $5 \mathrm{~m}$ test $(\mathrm{r}>0.62$, large-very large). Contrary to our results, Yanci et al. (2014b) found the same moderate relationship $(\mathrm{r}=0.53)$ between both acceleration tests (i.e. $5 \mathrm{~m}$ and $15 \mathrm{~m}$ ) and the MAT in young children (age $=7.6 \pm 0.8$ years). Thus, the issue of the relationships among the acceleration and CODA abilities in children remains unresolved. Even though some largevery large relationships have been described between some fitness tests, the association among motor abilities remains unclear not only for adults (Salaj and Markovic, 2011; Yanci et al., 2014a), but also for children (Yanci et al., 2014b). Moreover, it has been suggested that these motor abilities are independent (Salaj and Markovic, 2011). The reason may be found in the use of different tests to assess the same fitness variable, and the age and gender of the athletes (Vescovi and McGuigan, 2008; Yanci et al., 2014b). Among other factors, it would be interesting to know the impact of the maturation process (Malina et al., 2004) and the effects of training of each sport modality on these associations.

\section{Conclusions}

Our results show significant differences between U8-U10 and U12-U14 in the $5 \mathrm{~m}$ sprint and between U8-U10 and U10-U12 in CODA (505 and MAT). Moreover, the HCMJ and HCMJAS also differed between U8-U10, U10-U12 and between U12-U14. However, no significant differences between U14-U16 were observed in any of the physical tests. 
Considering the age and gender interaction, the results obtained in this study indicate that there are no differences between boys and girls in the physical performance level in U8 and U10 categories. However, in U12 and U14 categories, the boys showed better performance than the girls in the MAT. Horizontal jump performance was better in the U16 boys than in the U16 girls. Possibly gender differences in sprinting, CODA, vertical jumping and horizontal jumping do not occur until after the age of 10 years and thus, they might have a different outcome depending on the analyzed capacity.

\section{References}

Amusa LO, Goon DT, Amey AK. Gender differences in neuromotor fitness of rural South African children. Med Sport, 2010; 63: 221-37

Armstrong N, Welsman JR, Chia M. Short term power output in relation to growth and maturation. $\mathrm{Br} J$ Sports Med, 2001; 35: 118-124

Brughelli Cronin Levin Chaouachi A. Understanding change of direction ability in sport. Sports Med, 2008; 38(12): 1045-1063

Castro-Pinero J, Gonzalez-Montesinos JL, Keating XD, Mora J, Sjostrom M, Ruiz JR. Percentile values for running sprint field tests in children ages 6-17 years: influence of weight status. Res Q Exer Sport, 2010; 81(2): 143-151

Catley MJ, Tomkinson GR. Normative health-related fitness values for children: analysis of 85347 test results on 9-17-year-old Australians since 1985. Br J Sports Med, 2013; 47: 98-108

Chaouachi A, Manzi V, Chaalali A, Wong del P, Chamari K, Castagna C. Determinants analysis of changeof-direction ability in elite soccer players. J Strength Cond Res, 2012; 26(10): 2667-2676

Cohen J. Statistical power analysis for the behavioral sciences. Hillsdale, NY: LEA; 1988

Doré E, Bedu M, Van Praagh E. Squat jump performance during growth in both sexes: Comparison with cycling power. Res Q Exer Sport, 2008; 79(4): 517-524

Eisenmann JC, Malina RM. Age- and sex-associated variation in neuromuscular capacities of adolescent distance runners. J Sports Sci, 2003; 21(7): 551-557

Hoare DG, Warr CR. Talent identification and women's soccer: An Australian experience. J Sports Sci, 2000; 18(9): 751-758

Hopkins WG, Marshall SW, Batterham AM, Hanin J. Progressive statistics for studies in sports medicine and exercise science. Med Sci Sports Exerc, 2009; 41(1): 3-13

Jaric S, Ugarkovic D, Kukolj M. Evaluation of methods for normalizing muscle strength in elite and young athletes. J Sports Med Phys Fitness, 2002; 42(2): 41-151

Kugler F, Janshen L. Body position determines propulsive forces in accelerated running. J Biomech, 2010; 43: 343-348

Lam HM, Schiller W. A pilot study on the gross motor proficiency of Hong Kong preschoolers aged 5 to 6 years. Early Child Dev Care, 2001; 171(1): 11-20

Lätt E, Mäestu J, Ortega FB, Rääsk T, Jürimäe T, Jürimäe J. Vigorous physical activity rather than sedentary behaviour predicts overweight and obesity in pubertal boys: A 2-year follow-up study. Scand J Public Health, 2015; 43(3): 276-282

Lopes VP, Rodrigues LP, Maia JA, Malina RM. Motor coordination as predictor of physical activity in childhood. Scand J Med Sci Sports, 2011; 21(5): 663-669

Malina RM, Eisenmann JC, Cumming SP, Ribeiro B, Aroso J. Maturity-associated variation in the growth and functional capacities of youth football (soccer) players 13-15 years. Eur J Appl Phys, 2004; 91(5-6): $555-562$ 
Maulder P, Cronin J. Horizontal and vertical assessment: reliability, symmetry, discriminative and predictive ability. Phys Ther Sport, 2005; 6: 74-82

McKenzie TL, Sallis JF, Broyles SL, Zive MM, Nader PR, Berry CC, Brennan JJ. Childhood Movement Skills: Predictors of Physical Activity in Anglo American and Mexican American Adolescents? Res $Q$ Exerc Sport, 2002; 73: 238-244

Mendez-Villanueva A, Buchheit M, Kuitunen S, Douglas A, Peltola E, Bourdon P. Age-related differences in acceleration, maximum running speed, and repeated-sprint performance in young soccer players. $J$ Sports Sci, 2011; 29(5): 477-484

Mero A. Force-time characteristics and running velocity of male sprinters during the acceleration phase of sprinting. Res Q Exerc Sport, 1998; 59: 94-98

Meylan CM, Cronin JB, Oliver JL, Rumpf MC. Sex-related differences in explosive actions during late childhood. J Strength Cond Res, 2014; 28: 2097-2104

Ortega FB, Ruiz JR, Castillo MJ. Physical activity, physical fitness, and overweight in children and adolescents: evidence from epidemiologic studies. Endocrinol Nutr, 2013; 60(8): 458-469

Ortega FB, Cadenas-Sánchez C, Sánchez-Delgado G, Mora-González J, Martínez-Téllez B, Artero EG, CastroPiñero J, Labayen I, Chillón P, Löf M, Ruiz, JR. Systematic Review and Proposal of a Field-Based Physical Fitness-Test Battery in Preschool Children: The PREFIT Battery. Sports Med, 2015; 45(4): 533555

Salaj S, Markovic G. Specificity of jumping, sprinting, and quick change of direction motor abilities. J Strength Cond Res, 2011; 25(5): 1249-1255

Sekulic D, Spasic M, Mirkov D, Cavar M, Sattler T. Gender-specific influences of balance, speed, and power on agility performance. J Strength Cond Res, 2013; 27(3): 802-811

Sheppard JM, Young WB. Agility literature review: classifications, training and testing. J Sports Sci, 2006; 24(9): 919-932

Spencer M, Pyne D, Santisteban J, Mujika I. Fitness determinants of repeated-sprint ability in highly trained youth football players. Int J Sports Phys Perform, 2011; 6: 497-508

Taeymans J, Clarys P, Hassane A, Hebbelinck M, Duquet W. Developmental changes and predictability of static strength in individuals of different maturity: A 30-year longitudinal study. J Sports Sci, 2009; 27(8): 833-841

Vescovi JD, McGuigan MR. Relationships between sprinting, agility, and jump ability in female athletes. J Sports Sci, 2008; 25: 97-107

Vescovi JD, Rupf R, Brown TD, Marques MC. Physical performance characteristics of high-level female soccer players 12-21 years of age. Scand J Med Sci Sports, 2011; 21(5): 670-678

Yanci J, Reina R, Los Arcos A, Camara J. Effects of different contextual interference training programs on straight sprinting and agility performance of primary school students. J Sports Sci Med, 2013; 12(3): 601-607

Yanci J, Los Arcos A, Mendiguchia J, Brughelli M. Relationships between sprinting, agility, one- and two-leg vertical and horizontal jump in soccer players. Kinesiol, 2014a; 46(2): 194-201

Yanci J, Los Arcos A, Grande I, Gil E, Cámara J. Correlation between agility and sprint according to student age. Coll Antropol, 2014b; 38(2): 533-538

\section{Corresponding author:}

\section{Javier Yanci, PhD}

University of the Basque Country (UPV/EHU), Physical Education and Sports Department, Faculty of Education and Sport, University of The Basque Country, Lasarte s/n, 01007 Vitoria-Gasteiz, Spain.

Tel. 0034945013529

E-mail: javier.yanci@ehu.es 